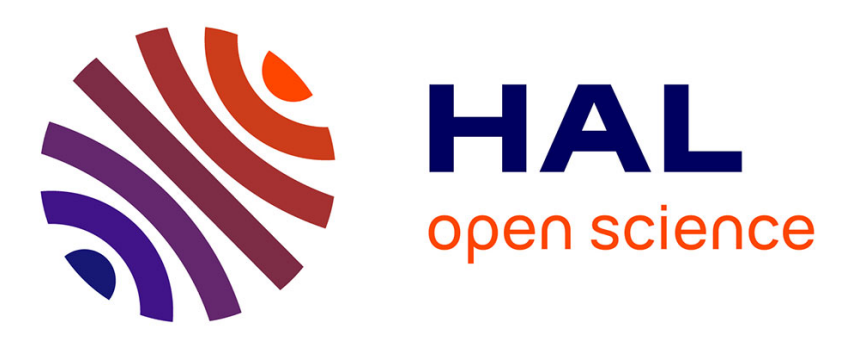

\title{
Karl Kraus contre l'école de Freud ou comment délégitimer l'interprétation psychanalytique de la littérature
}

Gerald Stieg, Jean-François Laplénie

\section{- To cite this version:}

Gerald Stieg, Jean-François Laplénie. Karl Kraus contre l'école de Freud ou comment délégitimer l'interprétation psychanalytique de la littérature. Savoirs et clinique - Revue de Psychanalyse, 2005, 6 (1), pp.53-58. 10.3917/sc.006.0053 . hal-02900655

\section{HAL Id: hal-02900655 \\ https: / hal.sorbonne-universite.fr/hal-02900655}

Submitted on 16 Jul 2020

HAL is a multi-disciplinary open access archive for the deposit and dissemination of scientific research documents, whether they are published or not. The documents may come from teaching and research institutions in France or abroad, or from public or private research centers.
L'archive ouverte pluridisciplinaire HAL, est destinée au dépôt et à la diffusion de documents scientifiques de niveau recherche, publiés ou non, émanant des établissements d'enseignement et de recherche français ou étrangers, des laboratoires publics ou privés. 


\title{
KARL KRAUS CONTRE L'ÉCOLE DE FREUD OU COMMENT DÉLÉGITIMER L'INTERPRÉTATION PSYCHANALYTIQUE DE LA LITTÉRATURE
}

\author{
Gérald Stieg, Jean-François Laplénie
}

ERES | «Savoirs et clinique »

2005/1 n 6 | pages 53 à 58

ISSN 1634-3298

ISBN 2749204259

\section{Article disponible en ligne à l'adresse :}

https://www.cairn.info/revue-savoirs-et-cliniques-2005-1-page-53.htm

Distribution électronique Cairn.info pour ERES.

(C) ERES. Tous droits réservés pour tous pays.

La reproduction ou représentation de cet article, notamment par photocopie, n'est autorisée que dans les limites des conditions générales d'utilisation du site ou, le cas échéant, des conditions générales de la licence souscrite par votre établissement. Toute autre reproduction ou représentation, en tout ou partie, sous quelque forme et de quelque manière que ce soit, est interdite sauf accord préalable et écrit de l'éditeur, en dehors des cas prévus par la législation en vigueur en France. Il est précisé que son stockage dans une base de données est également interdit. 


\title{
Karl Kraus contre l'école de Freud ou comment délégitimer l'interprétation psychanalytique de la littérature
}

\author{
érès | Savoirs et clinique \\ 2005/1 - no6 \\ pages 53 à 58 \\ ISSN 1634-3298
}

\section{Article disponible en ligne à l'adresse:}

http://www.cairn.info/revue-savoirs-et-cliniques-2005-1-page-53.htm

\section{Pour citer cet article :}

"Karl Kraus contre l'école de Freud ou comment délégitimer l'interprétation psychanalytique de la littérature", Savoirs et clinique, 2005/1 no6, p. 53-58.

Distribution électronique Cairn.info pour érès.

(c) érès. Tous droits réservés pour tous pays.

La reproduction ou représentation de cet article, notamment par photocopie, n'est autorisée que dans les limites des conditions générales d'utilisation du site ou, le cas échéant, des conditions générales de la licence souscrite par votre établissement. Toute autre reproduction ou représentation, en tout ou partie, sous quelque forme et de quelque manière que ce soit, est interdite sauf accord préalable et écrit de l'éditeur, en dehors des cas prévus par la législation en vigueur en France. II est précisé que son stockage dans une base de données est également interdit. 


\section{Karl Kraus contre l'école de Freud ou comment délégitimer I'interprétation psychanalytique de la littérature}

\section{Gerald Stieg Jean-François Laplénie}

Dans une réflexion sur le «culte des dictateurs » (Diktatorenverehrung) des années trente Robert Musil a dressé, en 1937, une liste aussi inattendue que remarquable d'intellectuels célèbres qui ont attiré sur leur personne «le culte intellectuel des dictateurs ou le culte des dictateurs intellectuels » (geistige Diktatorenverehrung ${ }^{1}$ ) qui aurait précédé le culte politique des Mussolini, Hitler et Staline. Dans cette liste sont associés les noms suivants : Stefan George, Kraus, Freud, Jung, Adler, Klages et Heidegger, donc la psychanalyse dans ses trois variantes principales. Elle se trouve ainsi en compagnie de l'un de ses détracteurs les plus radicaux et les plus spirituels. Musil ne parle pas des contenus, mais exclusivement d'une analogie structurelle qui aurait (qui a) pour dénominateur commun « le besoin de l'incarnation d'un sauveur», «la croyance irréligieuse » ou - ce qui est encore plus clair - «l'explication complète comme mauvais signe». Jacques Bouveresse commente cette attitude comme suit : «La fonction qu'ils (les intellectuels de type dictatorial) remplissent est celle de la croyance religieuse dans la version irréligieuse de celle-ci ${ }^{2}$. » Autrement dit, la réapparition de systèmes dogmatiques qui ont réponse à tout et sont immunisés contre toute critique.

Thomas Szasz, détracteur violent de la psychanalyse, a entrepris dans sa diatribe antifreudienne Karl Kraus et les docteurs de l'âme ${ }^{3}$ - visiblement sans connaître la position de Musil - une séparation manichéenne entre la

Gerald Stieg, professeur (Études germaniques) à la Sorbonne Nouvelle-Paris III, directeur de I'Institut d'allemand. Jean-François Laplénie, docteur de la Sorbonne Nouvelle, ATER à I'Institut d'allemand de la Sorbonne Nouvelle-Paris III. 1. Robert Musil, Tagebücher, Reinbek, Rowohlt, 1976, p. 896.

2. Jacques Bouveresse, L'homme probable. Robert Musil, le hasard, la moyenne et l'escargot de l'histoire, Combas, L'Éclat, 1993, p. 271.

3. Thomas Szasz, Karl Kraus et les docteurs de l'âme. Un pionnier et sa critique de la psychiatrie et de la psychanalyse, Paris, Hachette, 1985 (édition anglaise : Londres, Routledge and Kegan Paul, 1976). 
rhétorique « vile » de Freud (et de la psychanalyse en général) et la rhétorique «noble» de Karl Kraus qui est son témoin principal dans le procès qu'il intente à Freud. Dans son argumentation, il s'appuie sur Egon Friedell, Karl Popper, Karl Jaspers et Eric Voegelin pour appliquer le diagnostic de Musil exclusivement à Freud et, dans une moindre mesure, à Adler. La délégitimation de la psychanalyse va très loin : elle est traitée de «pseudo-religion juive» ou de « secte» (Friedell $\left.{ }^{4}\right)$, d' « astrologie » ou de «légende» $\left(\right.$ Popper ${ }^{5}$ ), enfin de «mouvement gnostique » (Voegelin). Parmi les « mouvements gnostiques modernes » dont le but est la soumission des êtres, Voegelin énumère entre autres « le marxisme, la psychanalyse, le communisme, le fascisme, et le national-socialisme ${ }^{6}$ ». Comme Szasz, mais contrairement à Musil dont il semble par ailleurs partager la perspective, Voegelin voit en Karl Kraus un véritable antidote à toutes les formes de gnose moderne. Appuyé sur ces autorités, Szasz arrive à une condamnation sans appel de la psychanalyse : elle est, selon lui, une «pratique collectiviste et coercitive du contrôle social ${ }^{7}$ ».

Comme par miracle, de la liste des vils rhéteurs de Musil qui auraient préparé les esprits à la dictature politique, le nom de Kraus n'est pas seulement retranché, mais transformé en unique «sauveur» des valeurs nobles et de la dignité humaine. Sans s'en rendre compte, Szasz fait, en effet, de Kraus un dictateur d'une « croyance religieuse dans sa version irréligieuse ». Il oublie une dimension de Kraus qu'Elias Canetti a mise au jour dans son essai particulièrement perspicace sur le satiriste Karl Kraus ou l'école de la résistance (1965), à savoir le «juge suprême » qui prononce des arrêts de mort et qui a su créer une "masse haineuse formée d'intellectuels» (intellektuelle Hetzmasse ${ }^{8}$ ).
Cette prise de distance de Canetti par rapport à Kraus, dont il avait été littéralement « l'esclave » des années durant, n'altère en rien son admiration pour les Derniers jours de l'humanité. Curieusement, Szasz ne fait aucune mention de l'opposition de Kraus à Freud que Canetti partage entièrement. Szasz classe les interprètes de Kraus selon un critère unique : partagent-ils ou non sa critique de la psychanalyse ? Quant à la dénonciation de l'habitus «dictatorial» de Kraus, Musil et Canetti sont du même avis, et ils ont sans doute raison. Kraus mérite amplement la place sur la liste de Musil en compagnie de Freud et de George.

Car Freud et Kraus agissent de façon presque identique dans leur champ respectif, et leur conflit s'explique largement par le fait indéniable que la nouvelle science psychanalytique (comme déjà avant elle la psychiatrie et la psychopathologie de type Lombroso ou les deux mélangées) commençait à quitter le territoire « scientifique » pour pénétrer dans le champ littéraire. Avant cette irruption qui sera bientôt suivie par l'engouement des écrivains pour la psychanalyse, Kraus et Freud ne sont nullement adversaires, au contraire. La Fackel est extrêmement favorable aux thèses psychanalytiques qui fournissent des arguments de poids aux attaques de Kraus contre la morale sexuelle hypocrite de l'avant-guerre. Freud et Kraus sont unis dans le combat contre une justice sexuelle aveugle. Freud lit la Fackel, Kraus écoute des cours de Freud à l'Université. La Fackel est même un outil apprécié pour faire connaître la psychanalyse dans le monde littéraire. Dans la lettre que Freud a adressée à Kraus le 18 novembre 1906, il y a, au-delà de l'admiration sincère pour le style et l'esprit (Witz) de Kraus, un véritable appel à la solidarité des «Wir wenigen»

\author{
4. Szasz, ibid., p. 100. \\ 5. Szasz, ibid., p. 103. \\ 6. Szasz, ibid., p. 108. \\ 7. Szasz, ibid., p. 109. \\ 8. Elias Canetti, Das Gewissen der Worte, Munich, Hanser, 1975, p. 41.
}


(« Nous, la petite minorité ») contre la grande majorité des bien-pensants qui se sentent «en sécurité » grâce à leur nombre. Cet appel de Freud est plus qu'une fleur rhétorique ou une flatterie à l'égard de l'homme qui occupait alors une position unique dans le monde des lettres. Il est évident que tous deux mènent un combat semblable, mais avec des moyens différents et sur des territoires primitivement séparés. Et il est indéniable que la pratique satirique de Kraus ressemble fondamentalement à une magistrale psychanalyse sociale. Le Malaise dans la culture de Freud et Troisième nuit de Walpurgis de Kraus sont plus proches l'un de l'autre que leurs auteurs ne pouvaient l'admettre.

Dans ce qui suit, l'accent sera mis presque exclusivement sur le combat pour la domination des territoires occupés par l'un ou l'autre. Dans ce combat, Freud a un comportement problématique : il impose au mouvement psychanalytique son propre silence dédaigneux à l'égard de Kraus et imite ainsi la tactique du «Totschweigen» (tuer par le silence) que la presse libérale viennoise a exercée face à la critique krausienne. Il faut bien dire que ce parallèle dans le comportement peut être interprété comme « un mauvais signe » de la part d'une puissance (consacrée ou naissante) qui décide de ne jamais répondre aux arguments de ses adversaires. Cela se comprendrait encore s'il s'agissait d'arguments en provenance du crétinisme alpin, mais les arguments de Kraus contre la presse, comme contre la psychanalyse, méritent à l'évidence attention, même si leur caractère ludique et excessif peut parfois cacher le sérieux de la démonstration.

La rupture entre Kraus et Freud est expliquée généralement par la malheureuse tentative de Fritz Wittels, ancien collaborateur de Kraus et propagateur d'idées freudiennes dans la $\mathrm{Fac}$ kel, d'entreprendre devant les membres de la Mittwochgesellschaft une psychanalyse semi- publique de Kraus sous le titre Die Fackelneurose, autrement dit de soumettre, contre sa volonté, un artiste vivant aux mécanismes explicatifs - Edipe en tête - de la psychanalyse. Ce n'est pas le cas individuel de Wittels qui nous intéresse ici (largement documenté par les travaux de Leo Lensing et Edward Timms, ainsi que par le catalogue du Schiller Nationalmuseum de $1999^{9}$ ), mais les conséquences de la transgression des champs entreprise par Wittels. Celui-ci se croyait probablement autorisé à ce genre de procédé, car le maître lui-même avait déjà donné l'exemple avec l'analyse du roman Gradiva de Gustav Jensen (1907), avec Der Dichter und das Phantasieren (1908) et Eine Kindheitserinnerung des Leonardo da Vinci (1910). De plus, il faut penser aux sous-produits psychanalytiques que furent les pathographies d'auteurs comme Kleist ou Lenau d'Isidor Sadger, ou l'analyse appliquée à Richard Wagner par le musicologue-psychanalyste Max Graf. Dès que les psychanalystes s'approchent du territoire de la littérature ou de l'art, Kraus réagit immédiatement en les accusant de pratiquer « une psychologie illégitime » (Unbefugte Psychologie $\left.{ }^{10}\right)$. Sa réaction à Max Graf (Richard Wagner im 'Fliegenden Holländer', paru en 1911 dans la collection «Schriften zur angewandten Seelenkunde» chez Deuticke, l'un des éditeurs de Freud) n'est pas seulement d'une drôlerie irrésistible mais relève également d'une perspicacité méthodologique qui sape la légitimité de la psychanalyse dans le domaine artistique :

«Une science qui en sait aussi peu sur le sexe que sur l'art répand le bruit que la sexualité du poète est 'sublimée' dans l'œuvre d'art. Une belle mission pour l'art que de faire l'économie du bordel ! Ce serait une bien meilleure mission pour le bordel que de faire l'économie de la sublimation par une œuvre d'art. À quel point ce procédé appliqué par les artistes est discutable,

9. Voir Thomas Szasz et surtout Edward Timms (éd.), Freud et la femme-enfant. Mémoires de Fritz Wittels. Suivi de Sigmund Freud, I'homme, la doctrine, l'école, Paris, PUF, 1999.

10. Die Facke/ 387/388, novembre 1913, p. 17-22. 
sans parler de son aspect peu économique, c'est ce que prouve le cas de ce grand musicien que la science en question aime à citer comme exemple de sublimation réussie. Les auditeurs de sa musique se sentent tellement excités par la sexualité qui y est sublimée, qu'il ne leur reste plus d'autre échappatoire que celle qu'a évitée l'artiste, sauf s'ils sont eux-mêmes capables de procéder à une nouvelle sublimation. Si l'artiste avait choisi le chemin le plus aisé, cet effet aurait été épargné à l'auditeur. C'est ainsi qu'à cause de la mauvaise habitude qu'ont les artistes de sublimer la sexualité, celle-ci est libérée, et qu'une affaire qui devrait demeurer une affaire privée de l'artiste, dégénère en scandale public ${ }^{11}$. » Mais Kraus ne se limite pas à cette demonstratio ad absurdum de la théorie de la sublimation. L'explication de la genèse du Vaisseau fantôme par le complexe d'Edipe inspire à Kraus le raisonnement suivant. Étant donné que le complexe d'Edipe est universel, le psychanalyste devrait chercher ce qui distingue psychologiquement Wagner des autres hommes : « Car Wagner est de tous les hommes le seul à qui peut être attribuée la paternité du 'Vaisseau fantôme', tandis que la plupart des autres qui avaient le désir mégalomane d'égaler leur père doivent à ce désir leur carrière de boursicoteur, avocat, conducteur de tramway ou critique musical, et seuls ceux qui avaient rêvé de devenir des héros sont devenus psychologues ${ }^{12}$. »

Et Kraus d'enfoncer le clou: "Car les scientifiques savent tout sauf l'essentiel, c'està-dire que tout ce qui concerne la sexualité et même l'onanisme est soumis à la règle du 'si duo faciunt'. Et que l'art est de toute façon non idem $^{13}$. » L'opposition entre «science» et «art» est absolument fondamentale dans la stratégie que Kraus développe contre la psychanalyse. Il interdit à cette « science » (dont il met intrinsèquement en cause le caractère vraiment « scientifique ») l'entrée sur son territoire, interdiction joliment exprimée par l'aphorisme : «Après mûre réflexion je préfère retourner au pays de l'enfance plutôt en compagnie de JeanPaul que de S. Freud ${ }^{14}$. »

L'âpreté de ce conflit, que Freud préfère refouler et passer sous silence, est particulièrement sensible lorsqu'il touche Goethe, l'icône culturelle par excellence dont Kraus et Freud partagent le culte. Un trait caractéristique de la démarche de Freud est la recherche d'une légitimité dans la «grande littérature » (tragédie grecque, Shakespeare, Goethe, Schiller, Heine, Dostoïevski). C'est à cause de ce besoin de légitimité qu'il faisait même semblant d'adhérer au mythe personnel de C.G. Jung qui se voulait un descendant illégitime de Goethe. Quant à Kraus, il se considérait ouvertement comme le gardien du temple de la tradition goethéenne et sévissait contre toute déformation des textes sacrés, notamment du Chant nocturne du voyageur. Il ne faut surtout pas oublier que son règlement de compte avec le national-socialisme, à propos duquel il dénonce les abus commis par Spengler et consorts, se fait au nom et à l'aide du «Faust ». C'est dans cette optique qu'il faut voir sa réaction aux « interprétations » psychanalytiques de la ballade de «L'apprenti sorcier» qui hésitaient entre l'incontinence nocturne et la masturbation. Cette attaque contre la «psychologie illégitime ${ }^{15} »$ se dirige plutôt contre les « apprentis sorciers » à la Sadger et Wittels que contre le vieux « maître sorcier » lui-même. De toute façon, Kraus retourne avec un malin plaisir l'interprétation psychanalytique du poème contre les interprètes eux-mêmes.

Thomas Szasz a accompagné sa philippique antipsychanalytique d'une anthologie de

11. Die Fackel 406/412, octobre 1915, p. 132-133.

12. Die Fackel 376/377, le 30 mai 1913, p. 21.

13. Die Fackel 381/383, septembre 1913, p. 74.

14. Ibid., p. 73.

15. Ibid., p. 74. 
soixante-dix pages qui contient la plupart des textes (aphorismes, poèmes, essais) que Kraus a consacrés à la psychanalyse. Et c'est une lecture fort plaisante.

Notre approche du conflit est exclusivement centrée sur la confusion des champs littéraire et scientifique. Kraus est un «puriste» absolu. Dès le début de la Fackel, il s'agissait pour lui de séparer radicalement la sphère artistique de la sphère journalistique et de combattre toute forme de contamination entre les deux. Or, dans une certaine mesure, l'apparition de la psychanalyse dans le champ littéraire, souvent relayée par la presse, provoque chez lui un rejet comparable à celui du journalisme. Un reproche souvent dirigé contre le journalisme, l'âpreté au gain, est également appliqué à la psychanalyse (souvent avec une touche antisémite comme chez Friedell). Un seul exemple suffit : "Ils mettent leur main dans notre rêve comme s'il s'agissait de notre porte-monnaie ${ }^{16}$. »

Kraus a vite compris que la première conférence que Freud a tenue en dehors du Allgemeines Krankenhaus était un véritable acte fondateur pour le rapprochement de la sphère «scientifique » et de la sphère littéraire et artistique. Il s'agissait de la conférence «Der Dichter und das Phantasieren » (L'écrivain et le rêve éveillé) qui a eu lieu le 6 décembre 1907 devant un public trié sur le volet dans la librairie Hugo Heller, haut lieu de la culture viennoise où des célébrités comme Thomas Mann ou Rilke faisaient des lectures publiques et où des peintres comme Kokoschka exposaient leurs tableaux. Un acte manqué (lapsus calami) de Freud illustre à merveille l'importance qu'il attribuait lui-même à cette incursion dans le monde des lettres. Il écrit à Jung à propos de cette conférence devant «environ 90 personnes » : « ... pour de nombreux poètes et leur dame ç'aura été un lourd repas. Dans l'ensemble c'était seulement un hors-d'œuvre pour donner de l'appétit. La Neue Rundschau s'est assurée la conférence à l'état fœtal, elle y sera probablement reproduite ${ }^{17}$. » Or la conférence a été reproduite non pas dans la Neue Rundschau, mais dans un organe peu connu, la Neue Revue (Berlin). Visiblement, Freud a pris ici son désir - l'entrée dans la revue littéraire la plus prestigieuse d'Allemagne - pour une réalité nettement moins glorieuse. Néanmoins, le pas en direction du champ «culturel » est accompli avec succès, et Hugo Heller sera même l'un des éditeurs de Freud, notamment de la Gradiva (1907) et de la revue Imago (de 1912 à 1918). Mutatis mutandis, on peut comparer le passage de la psychanalyse à la maison d'édition Heller avec le choix de Sartre de publier ses œuvres philosophiques chez Gallimard, éditeur littéraire.

Karl Kraus ne manquera aucune occasion pour stigmatiser la confusion des genres qui fait le succès culturel de Heller et, pratiquement toujours, il y associe la psychanalyse, notamment dans la satire littéraire Littérature ou dans l'adaptation des Oiseaux d'Aristophane sous le titre Wolkenkuckucksheim. Les satires littéraires de Kraus, auxquelles il faut ajouter les pièces Traumstück et Traumtheater qui traitent largement de la relation entre littérature et psychanalyse, sont des témoignages précieux de la pénétration de la psychanalyse dans le monde littéraire et dans le code culturel en général. Kraus y voit la transformation illégitime d'une «science de l'âme non autorisée » en phénomène de mode, voire en commerce douteux.

Malgré l'acharnement de Kraus contre les apprentis sorciers de la psychanalyse et le silence dédaigneux du vieux maître sorcier, il faut se rendre à l'évidence qu'il existe une parenté fondamentale entre leurs méthodes, car le champ de bataille est la langue et son usage (notamment littéraire); et la division se fera sur 
le principe qui l'anime, c'est-à-dire «volonté créatrice » contre « inconscient ». D'où le renversement systématique des prétentions de la psychanalyse par Kraus. Par exemple : «Je ne sous-estime certainement pas la valeur de l'étude scientifique de la vie sexuelle. Cela reste tout de même une belle tâche. Et lorsque ses résultats sont confirmés par les conclusions de l'imagination artistique, cela est flatteur pour la science et elle n' aura pas vécu pour rien ${ }^{18}$. » $\mathrm{Ou}$ de façon plus lapidaire encore : «Mon inconscient connaît bien mieux le conscient d'un psychologue que le conscient de celui-ci ne connaît mon inconscient ${ }^{19}$. »

On voit bien à quel point la discorde peut naître sur fond d'une communauté de vue, dont Kraus est du reste parfaitement conscient : «On m'a souvent dit que certaines choses que j'ai trouvées sans les chercher devaient être vraies, puisque F. aussi les avait cherchées et trouvées. Une telle vérité serait sans doute un piètre critère. Car ce n'est que pour celui qui cherche que le but est important. Pour celui qui trouve, en revanche, c'est le chemin. Tous deux ne se rencontrent pas. L'un chemine un peu plus vite que l'autre n'arrive au but. Quelque chose leur est commun. Mais le prophète précède toujours le cavalier de l'Apocalypse et annonce sa venue $^{20}$. »

Ainsi l'affinité, le parallélisme, les ressemblances entre les deux méthodes ne seraient qu'apparents - ou plutôt seraient le résultat d'une erreur de perspective. L'écrivain ne cherche pas à accroître le savoir par des résultats, mais à parcourir - et sans doute faire parcourir un chemin qui y mène. Car si le but du voyage était le même, le chemin parcouru serait diamétralement opposé, et, par là même, la signification du voyage bouleversée. Ce qui est en jeu ici n'est ni l'objet ni même le résultat de la connaissance mais, pour parler avec Spinoza, le genre même de la connaissance impliquée. Kraus fait le diagnostic d'une disparité radicale entre le genre scientifique et celui qui relève de la littérature.

N.B. Cette communication est le fruit d'un travail à quatre mains de Gerald Stieg et de Jean-François Laplénie, dont la thèse "Puissances voisines. Études pour une histoire structurelle de la réception de la psychanalyse dans les milieux littéraires de langue allemande» (Paris III) a fourni les bases. 